\title{
RITMOS TEMPORALES Y DINÁMICAS DEL PAISAJE DE LA ALTA ALPUJARRA GRANADINA MEDIANTE SISTEMAS DE INFORMACIÓN GEOGRÁFICA*
}

\author{
$M^{\mathrm{a}}$ T. CAMACHO OLMEDO \\ Instituto de Desarrollo Regional, C/ Rector López Argüeta s/n, 18071 \\ Universidad de Granada, camacho@ugr.es
}

\begin{abstract}
Resumen: En las últimas décadas hemos asistido a una profunda reestructuración del paisaje de las áreas montañosas mediterráneas. En la Alta Alpujarra granadina, comarca localizada en la cara sur de Sierra Nevada, el paisaje ha sufrido las consecuencias del abandono de la agricultura de regadío en bancales y la lenta progresión de las comunidades de vegetación natural en estos espacios, mientras que, por otra parte, las campanas de repoblación forestal han transformado las laderas medias, lo que se une al avance natural de las formaciones arbóreas de encinares y robledales.
\end{abstract}

Con ayuda de un Sistema de Información Geográfica, se ha elaborado una serie cronológica de mapas de ocupación del suelo de la segunda mitad del siglo XX, donde la leyenda recoge con detalle las situaciones más complejas, especialmente aquellas ligadas a la transición entre categorías y a los paisajes en mosaico. Esta serie es la base del análisis espacio-temporal realizado mediante las funciones propias de un SIG para precisar las principales dinámicas, los titmos de las mismas y el balance y diagnóstico global de las transformaciones acaecidas.

Palabras clave: Paisaje, usos del suelo, dinámica, ritmos temporales, Sistemas de Información Geográfica

\begin{abstract}
In the lasts decades the mediterraneans mountains landscapes have been reorganized. In the region named Alta Alpujarra, the landscape has been afected by the agricultural's abandon and the vegetation communities progression in theses places. In the other hand, the pine's reforestation has changed the middle mountain and the oaks and ilex's communities advances in a natural process.
\end{abstract}

* Recibido: 10-11-03. Aceptado: 06-04-05.

La cartografía en color se incluye en un CD. 


\begin{abstract}
A cronological serie of land use maps in the second half of XX century has been builded with a Geographical Information System, where the legend includes the more complex situations, specially those from the transitions between categories and from the mosaic landscape. This serie is the first step for the spatial and temporal analysis, made by the fonctions of the GIS, to show the principals dynamics, the temporal rhythms and the globals changes diagnostics.
\end{abstract}

Key words: Landscape, land uses, dynamics, temporal rhythms, Geographic Information Systems

\title{
1. Introducción
}

El análisis de los cambios de usos del suelo y de coberturas constituye uno de los temas centrales de investigación cuando se intenta comprender el estado actual de nuestros paisajes y su proyección futura. Aunque de larga y dilatada trayectoria, este tema ha sido retomado con fuerza en la última década, convirtiéndolo en objeto de estudio propio y en el lema principal de grupos de trabajo en organismos como la Unión Geográfica Internacional (Land Use and cover change, IGU LUCC, descle 1996) o en congresos internacionales (Singh et al., 2001).

En esta línea de trabajo, la necesidad de trabajar con una cantidad ingente de información estadística y sobre todo espacial, ha motivado la utilización de herramientas como los Sistemas de Información Geográfica para poder trazar los principales ejes de los cambios en el paisaje. La versatilidad y adecuación de los SIG para el análisis espacio-temporal ha quedado manifiesta desde sus inicios, percibiéndose en los últimos años un creciente interés por explotar estas posibilidades de aplicación (Eastman et al., 1995).

En la bibliografía española son numerosas, desde principios de los años noventa, las contribuciones en las que se aplican las funciones propias de los Sistemas de Información Geográfica para el estudio evolutivo de los paisajes y de los usos del suelo (a modo de ejemplo, citaremos, entre otros, los trabajos de Castro y García, 1993; Comas et al, 1992; Martínez, J. y Romero, R., 1999; Otero, 1993; Otero, 1999; Palacios, 1994; Peña, en prensa; Sancho, Bosque y Moreno, 1993a; Sancho, Bosque y Moreno, 1993b). Los objetivos, técnicas y sofwares empleados en estos y otros trabajos presentan una alta variedad, oscilando sus resultados entre el diagnóstico descriptivo de los usos del suelo y los paisajes, el análisis de la estructura paisajística o la valoración de la calidad y fragilidad, aspectos todos ellos abordados según la dinámica temporal.

El trabajo que aquí presentamos debe enmarcarse en esta amplia línea de investigación, de retomada actualidad, y, en particular, en la metodología desarrollada en una 
serie de proyectos de investigación ${ }^{1}$. En ellos se han venido aplicando diversos métodos de modelización para trazar la dinámica de los paisajes de dos áreas montañosas mediterráneas. Los resultados que se exponen a continuación se centran en el análisis y explotación de la bases de datos espacio-temporal de una de estas áreas, la Alta Alpujarra granadina, particularmente afectada por la reestructuración que ha sufrido la montaña mediterránea en la segunda mitad del siglo XX, con el objetivo de detectar las principales transformaciones y poder analizar los ritmos temporales de dicha evolución.

La serie cronológica de los mapas de uso del suelo y vegetación desde 1957 hasta la actualidad, como componente que mejor recoge la caracterización fisonómica del paisaje, ha sido tratada con el Sistema de Información Geográfica Idrisi 32 Release 2 (Eastman, 2001), herramienta hoy día imprescindible para manejar y analizar bases de datos de gran volumen. Funciones propias de los SIG, como la superposición de capas temporales y la consulta condicional, han permitido obtener las cartografías evolutivas y las estadísticas resultantes y, a partir de ellas, concretar y trazar las dinámicas más significativas, los ritmos temporales de los períodos considerados y el balance global de los cambios. Este sistema de análisis, ya utilizado en otros trabajos afines (Martínez, Martín y Román, 2000, entre otros), es aquí esencial para poder manejar la amplia y compleja base de datos elaborada, que constituye, sin duda, la principal aportación de esta investigación.

En concreto, los objetivos planteados serian los siguientes:

- Trazar una serie cronológica de los usos del suelo y la vegetación del área de estudio para caracterizar el paisaje desde el punto de vista fisionómico.

- Aplicar las funciones propias de los SIG para el análisis espacio-temporal.

- Obtener las cartografías evolutivas por períodos y sus datos estadísticos, a fin de discriminar las diferentes etapas en el cambio de los paisajes y conocer sus ritmos temporales.

- Establecer el balance global de los cambios del paisaje.

- Analizar la síntesis de los cambios, precisando el número de transformaciones acaecidas y las dinámicas y tendencias más significativas, comparándolas con las áreas que muestran estabilidad.

\section{Presentación: Serie cronológica de los usos del suelo y vegetación de la Alta Alpujarra}

La disponibilidad de una serie cronológica de fotogramas aéreos y cartografía temática del área de estudio (vuelo americano de 1957, Mapa de Cultivos y Aprovechamientos del M.A.P.A. de 1974, y fotogramas de 1985 y de 1998) y la exis- 
tencia de un trabajo previo de interpretación de los fotogramas de 1985 (Jiménez, 1991), han facilitado la elaboración de los mapas de usos del suelo y vegetación, que, en virtud de la actualización efectuada en los trabajos de campo tras la fase de fotointerpretación, han sido identificados con la situación de la Alta Alpujarra en 1957, 1974, 1987 (actualización de la información de 1985), y 2001 (actualización de 1998).

La leyenda de la serie cronológica responde en lo esencial a la elaborada por Jiménez (1991) para el conjunto de Sierra Nevada. No obstante, para poder plasmar más adecuadamente los cambios en el paisaje, la dinámica interna y lá complejidad de las categorías a lo largo del período analizado, ha sido modificada y ampliada desde las 31 unidades iniciales hasta un total de 53. La Tabla 1 muestra la leyenda completa y la superficie (Has) que ocupa cada categoría en las fechas analizacias según el proceso de fotointerpretación.

Esta extensa leyenda recoge la diferenciación fisonómica de los usos del suelo y vegetación con un gran número de subunidades (en función, por ejemplo, del grado de cobertura), y amplía las unidades mixtas (mezcla de diferentes formaciones, especies o unidades en mosaico). Cabe destacar la identificaciôn de los tipos de matorral en función de las series de vegetación potencial y de los pisos bioclimáticos en los que se localizan, así como de su mayor o menor grado de cobertura vegetal, y la complejidad de las áreas abandonadas y semi-abandonadas. En este sentido debemos recordar que en la configuración definitiva del listado de categorías ha jugado un papel fundamental la información extraída del trabajo de campo, que enriquece y aporta un buen número de matices al planteamiento inicial.

Las 53 categorías pueden reagruparse en nueve clases que, en el último apartado de este trabajo, nos han servido para sintetizar los cambios y destacar las principales tendencias evolutivas. Siguiendo el orden de la leyenda en la Tabla 1, estas clases corresponderían a formaciones arbóreas (1-14), malorrales (15-25), pastizales (26-27), pinares de repoblación (28-31), cultivos abandonados (32-35), mosaico de cultivos abandonados y no abandonados (36-38), cultivos de regadio (39-43), cultivos de secano (44-47), mosaico de cultivos de regadio y secano (48-50) y uso urbano, minas $y$ canteras, $y$ ramblas (51-53).

\section{Las principales dinámicas de cambio por períodos (1957-74, 1974-87, 1987-2001)}

La superposición de los mapas de usos del suelo y vegetación por períodos muestra las áreas estables y las áreas que, por el contrario, han sufrido modificaciones. En concreto, se han contabilizado 149 dinámicas distintas entre 1957 y 1974,98 entre 1974 
y 1987 y 56 entre 1987 y 2001. De entre ellas, se han escogido por períodos las dinámicas más importantes en función de la superficie afectada (Tabla 2). Para su identificación, se especifica el tipo de cambio y se indica entre paréntesis la antigua y la nueva categoría según el orden que presentan las mismas en la leyenda de la Tabla 1.

Entre otras conclusiones, se puede indicar que el período de mayor transformación del paisaje en la Alta Alpujarra es el que oscila entre 1957 y 1974. Entre estas dos fechas se contabilizan 5086.07 hectáreas que han sufrido un cambio de categoría, cifra muy superior a la del período 1974-1987 (3494.68 hectáreas), o a las 3723.08 hectáreas que muestran una dinámica de cambio desde esta última fecha hasta 2001. En porcentajes, del total de las transformaciones observadas por periodos, el $41.34 \%$ se producen entre 1957 y 1974, el $28.40 \%$ entre 1974 y 1987, y el 30.26\% entre 1987 y 2001.

No solamente es mayor la intensidad del cambio en el período que oscila entre 1957 y 1974, sino que, como comentamos anteriormente, se observa también una mayor diversidad de dinámicas, descendiendo progresivamente esta cifra hasta el período más reciente. Además, si relacionamos el número de dinámicas y el total de la superficie transformada, en el primer periodo se constata que la superficie media por dinámica es de 34.13 hectáreas, inferior a la de 35.66 de $1974-87$, pero, sobre todo, muy alejada de las 66.48 hectáreas de media por dinámica entre 1987 y 2001, lo que indica una progresión hacia una mayor homogeneidad y concentración en los cambios.

Por otra parte, el peso de las principales dinámicas en el total de ellas varía según el período considerado. Entre 1957 y 1974, de las 149, las 13 más importantes representan un 54.44\% del total de "áreas dinámicas", cifra que aumenta al 69.35\% en el período de 1974 a 1987 para las 16 dinámicas más importantes, y al 82.81\% entre 1987 y 2001. Esta evolución confirma la tendencia hacia la menor dispersión y heterogeneidad en los cambios. Efectivamente, a medida que se avanza hacia fechas más recientes, la superficie transformada presenta una menor diversidad absoluta y relativa, al disminuir el número de dinámicas y aumentar el peso proporcional de las más importantes.

El análisis de la naturaleza de las transformaciones puede también afianzar esta tendencia. A modo ilustrativo, las figuras 1, 2 y 3 muestran, del total de dinámicas, las diez más importantes según la superficie afectada (ordenadas en este caso de mayor a menor superficie) para los períodos considerados. Observando estas figuras y los datos de la Tabla 2, se puede indicar que, entre 1957 y 1974, las dinámicas muestran un amplio abanico temático, oscilando entre la progresión de las formaciones de vegetación natural (especialmente de las masas de robledales y del piornal sobre áreas abandonadas) y las transformaciones de los cultivos de secano de las laderas más bajas, que tienden al monocultivo arbóreo. No obstante, las dinámicas más significativas están relacionadas con la intensa repoblación con pinares de este período y con el proceso de abandono de los cultivos de regadío y secano en altura. 


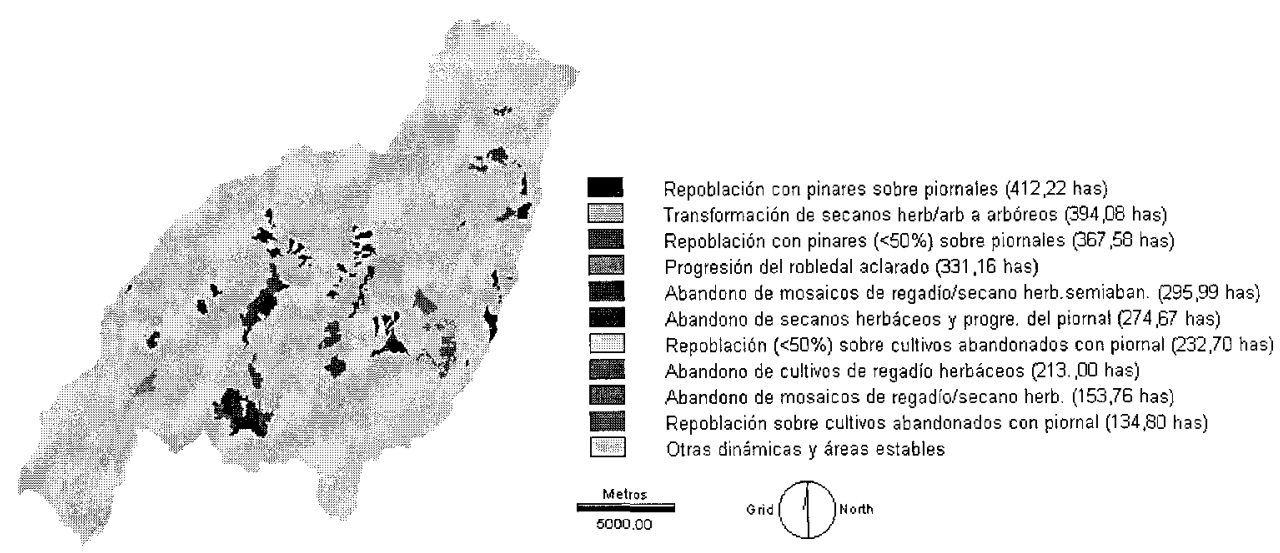

Figura 1. Principales dinámicas entre 1957-74 (Has). Fuente: Elaboración propia.

Entre 1974 y 1987 las transformaciones más importantes se pueden agrupar en torno a varios procesos concretos. Por un lado, la progresión y la regresión de las formaciones de vegetación natural, principalmente arbóreas, afectan a una amplia superficie, siendo poco significativa en este período la superficie repoblada con coníferas. Frente a esta dinámica natural, el abandono de los cultivos de regadío y secano sigue avanzando, aunque afecta ahora a las laderas medias, y se inicia el semi-abandono de los situados en las laderas más bajas cercanas a los núcleos urbanos.

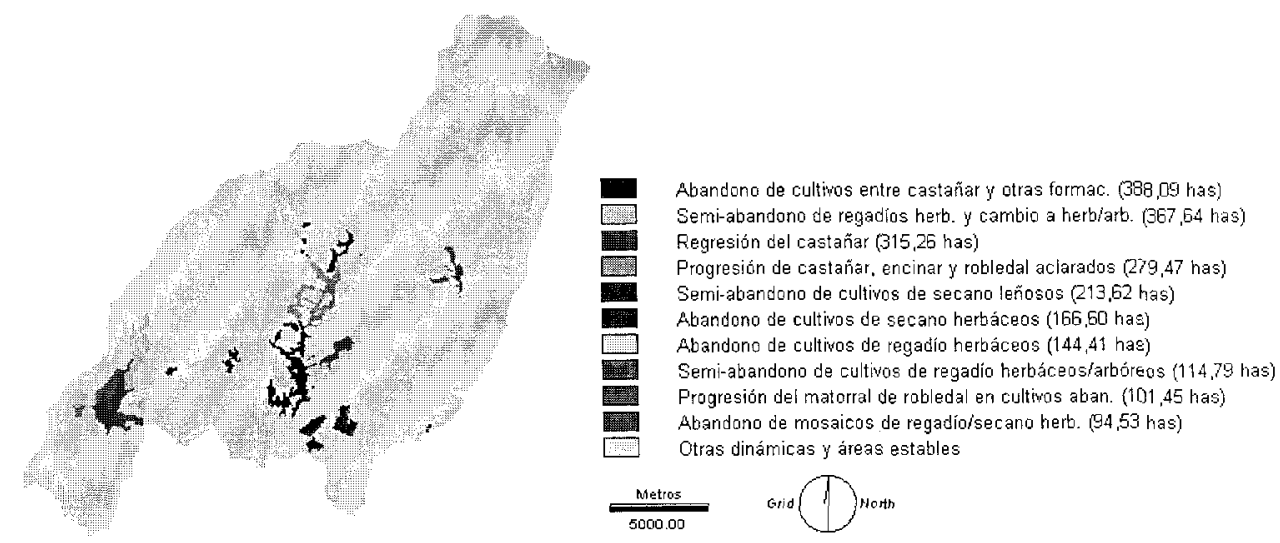

Figura 2. Principales dinámicas entre 1974- 1987 (Has). Fuente: Elaboración propia. 
Por último, entre 1987 y 2001, los cambios se centran, por un lado, en la dinámica de los pinares de repoblación (aunque la superficie repoblada en este periodo se limite a unas pocas hectáreas) y de las formaciones naturales arbóreas o arbustivas, y, por otro, en el proceso de abandono de cultivos y, sobre todo, de semi-abandono. Es esta última tendencia la que explica los principales cambios de usos del suelo y la que reagrupa la mayor superficie que sufre una transformación en este período, confirmando la tendencia hacia la concentración y homogeneización de las dinámicas.

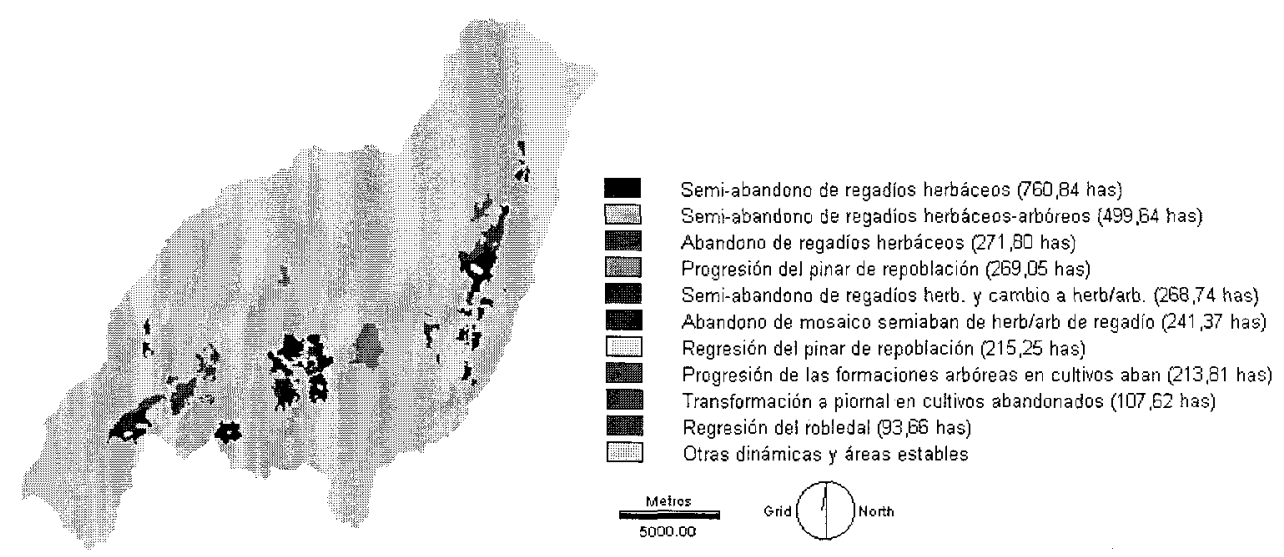

Figura 3. Principales dinámicas entre 1987-2001 (Has).

Fuente: Elaboración propia.

\section{Balance de los cambios y principales dinámicas entre 1957 y 2001}

La superposición del mapa de usos del suelo y vegetación más antiguo (1957) y más reciente (2001) de la serie cronológica, muestra el balance de los cambios acaecidos en el período considerado. De un total de 208 dinámicas contabilizadas, se muestran en la Tabla 3 las más significativas en función de la superficie afectada, indicándose, como en la Tabla 2, el tipo de cambio por categorías.

Hay que señalar que, al tratarse de la superposición entre dos fechas y no entre las cuatro de la serie, las cifras resultantes de cada dinámica no coinciden con las que podrían obtenerse por la suma por períodos de la Tabla 2. De ahí proviene el desajuste entre las 10178.43 hectáreas y 208 dinámicas obtenidas del balance global, y las 12303.83 hectáreas y 303 dinámicas obtenidas de la suma por períodos. Este hecho indica que han existido 95 tipos de transformaciones y 2125.4 hectáreas afectadas que no han dejado huellas en el paisaje en el año 2001. 
De los datos de la Tabla 3 puede constatarse que el peso de las principales transformaciones en el balance global de los cambios, si bien es inferior al observado en el período de 1987-01 y ligeramente superior al de 1957-74, es, no obstante, significativo. Así, las 14 dinámicas principales representan el $60.43 \%$ de las áreas dinámicas, mientras que la suma de las 194 dinámicas menores supone tan sólo el 39.56\% de las mismas. Poniendo en relación el número de cambios y la superficie, las dinámicas mayores reciben un valor medio de 439.35 hectáreas, mientras que las restantes dinámicas presentan un valor de tan sólo 20.76 hectáreas por dinámica. Todo ello indica una concentración en la evolución global de los usos del suelo y vegetación, aunque sea más manifiesta en las ûltimas décadas.

Las mismas dinámicas, ordenadas de mayor a menor superficie, se cartografían en la Figura 4. Agrupándolas por tipos de cambio y ordenándolas según la superficie afectada, destaca, en primer lugar, el proceso de abandono de los cultivos berbáceos, tanto de secano como de regadío, y la posterior progresión de los piornales o de las formaciones arbóreas en los campos abandonados.

En este sentido, se evidencia un generalizado proceso de "matorralización" de las áreas abandonadas (sobre todo por el piornal oromediterráneo que gana terreno a los antiguos cultivos de altura), unido a la invasión de especies arbóreas autóctonas de la zona, especialmente encinas, robles y castaños. Tan sólo en algunos casos la presencia de estas especies arbóreas era ya evidente en el período de actividad agrícola, siendo por lo tanto un proceso independiente al abandono, aunque se pueda ver favorecido tras él. Esta progresión vegetal es en algunos casos tan intensa que hace reintegrarse a los campos abandonados en las comunidades de vegetación natural propias de su serie potencial.

Concretando el proceso de abandono por tipos de cultivos, se puede decir que el secano herbáceo es prácticamente residual en la actualidad, no pudiendo reconocerse extensiones de tierras ocupadas exclusivamente por esta agricultura, sino sólo áreas en mosaico donde las parcelas de herbáceos de secano coexisten junto a herbáceos de regadío o bien configuran un mosaico de parcelas abandonadas junto a otras todavía activas. El abandono del secano herbáceo se produce fundamentalmente en el período que iría desde 1957 a 1974 y afecta a las parcelas situadas a mayor altitud en los valles. Este hecho provoca que no exista una sustitución de esta agricultura por otras especies leñosas, como ocurrió a mediados del siglo XIX al sustituirse los herbáceos por el viñedo, sino que se asiste simplemente a un manifiesto proceso de abandono y "erialización". Tan sólo algunas parcelas más accesibles y a menor altitud muestran una reconversión del secano herbáceo hacia el secano arbóreo (principalmente almendros) en los años setenta y ochenta del siglo XX.

En paralelo al proceso vivido por los cultivos de secano, el regadío sufre un grave proceso de abandono que hay que enmarcar en la crisis de la economía tradicional 
de montaña. Aunque esta agricultura contabiliza en el año 2001 más de 2000 hectáreas (Camacho et al, 2002a), sin embargo hay que subrayar que en las últimas cuatro décadas se ha reducido esta superficie a menos de la mitad de la existente en el año 1957. El abandono generalizado afecta fundamentalmente a los regadios eventuales o no permanentes, localizados a mayores altitudes y a más distancia de las poblaciones, quedando hoy día restringida esta agricultura a las vegas que circundan los núcleos urbanos. La relación entre el proceso de abandono y factores como la accesibilidad o la altitud (Camacho y Menor, 1997) explican la sucesión espacial desde 1957 y 2001 de los cultivos abandonados en la Alta Alpujarra.

Pero en la evolución del regadío es de destacar un proceso específico, que hemos denominado "semi-abandono" y que constituye otra de las grandes tendencias detectadas en la Figura 4. Las áreas semi-abandonadas corresponden o bien a mosaicos de parcelas abandonadas y no abandonadas, o bien a espacios infrautilizados en donde la explotación es mucho menos intensa que en épocas pasadas, aunque sin haberse llegado a una situación de abandono absoluto. El semi-abandono afecta tan sólo a los cultivos de regadío, ya sean herbáceos o, en menor medida, arbóreos, aunque se observa una tendencia hacia el mosaico de especies.

Se trata de un proceso reciente, que se inicia con posterioridad al abandono definitivo de las tierras más altas y que afecta en la actualidad también a las parcelas de mayor accesibilidad y cercanas a los núcleos de población. Las parcelas semi-abandonadas hacen acto de presencia en la cartografia evolutiva principalmente en el período que oscila entre 1974 y 1987, como fase de transición hasta el abandono generalizado de las parcelas, más evidente ya en el 2001.

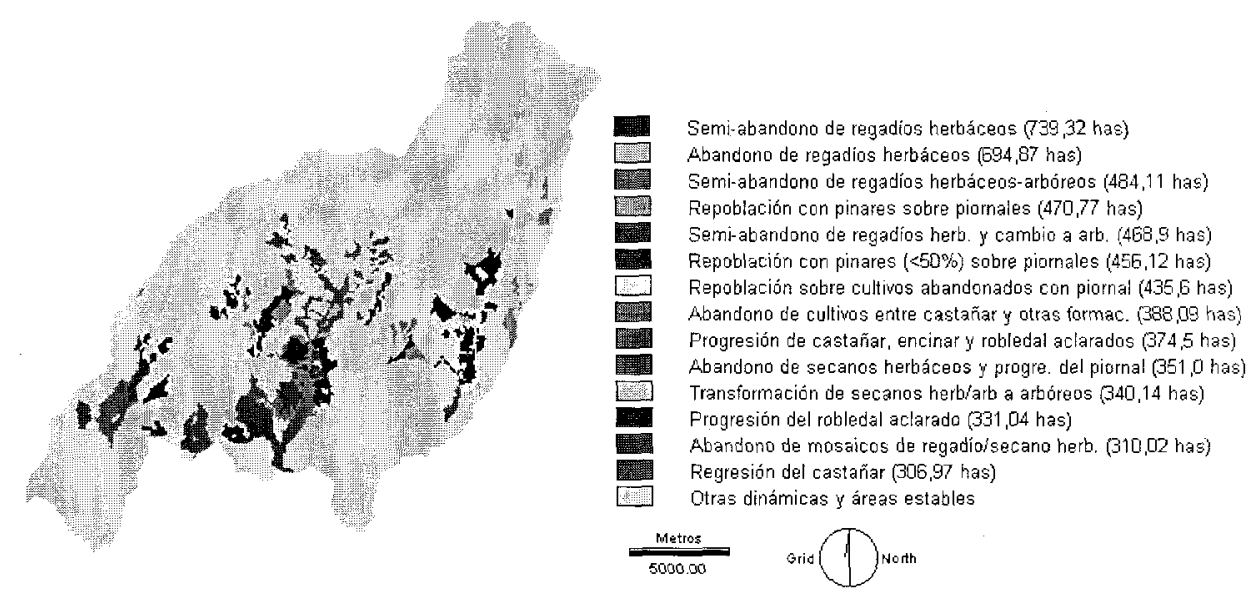

Figura 4. Principales dinámicas entre 1957-2001 (Has). Fuente: Elaboración propia. 
El tercer proceso a destacar es la repoblación con coniferas, con mayor o menor densidad de ocupación, que afecta fundamentalmente al piornal oromediterráneo, en algunos casos constituido tras la ocupación de las áreas abandonadas. Sin duda, uno de los procesos más evidentes de la transformación del paisaje de la Alta Alpujarra en el curso del siglo XX, generalizado por otra parte en el conjunto de las laderas medias que rodean el macizo de Sierra Nevada, ha sido esta campaña de repoblación forestal con coníferas por debajo de los 2500 metros de altitud, que ha dado lugar a una banda u orla casi continua de estas masas arboladas que, sin ser autóctonas, forman parte hoy día del paisaje "cotidiano" de este ámbito montañoso.

La cartografía evolutiva de las áreas repobladas en la segunda mitad del siglo XX (Camacho et al., 2002b) muestra que si bien en 1956 ya existía una amplia superficie afectada por esta tarea de reforestación (1482.40 has), iniciada a finales de los años veinte, es en el intervalo de tiempo que transcurre desde esta fecha hasta 1974 cuando se produce el mayor esfuerzo para proteger las laderas de estos municipios, llegando a alcanzarse una superficie total de 2996.41 has repobladas en esta fecha. Desde este momento hasta nuestros días, tan sólo se repueblan algunas parcelas que no modifican en demasía el paisaje forestal de la comarca, sumando un total de 3192.39 has repobladas en 1987 y 3224.13 en 2001. Estas nuevas áreas repobladas se restringen a parcelas de escasa superficie situadas a gran altitud, junto a otras masas arboladas o en aquéllas zonas afectadas por incendios en fechas anteriores.

Otros procesos de cambio dentro de las dinámicas principales se identifican con la progresión de las masas arbóreas (robledales, encinares y castañares) desde formaciones más aclaradas, y, en un enclave concreto del área de estudio, la regresión del castañar, que aparece ligada al proceso generalizado del abandono de la agricultura (Jiménez, 1991).

Aunque, efectivamente, las formaciones arbóreas de la Alta Alpujarra presentan una gran estabilidad e incluso una cierta progresión desde 1957 hasta nuestros días (Camacho et al., 2002b), esta tendencia general debe ser matizada según el tipo de especie. La citada progresión de la masa arbórea mixta de robles, encinas castaños, responde más bien a la evolución de una especie como la encina, mientras que sin embargo el castaño se muestra muy empobrecido y en franca regresión, salvo en aquellos enclaves en donde se beneficia de la proximidad de los cursos de agua o de las condiciones térmicas de la umbría. De ahí resultan las 374.5 hectáreas correspondientes a la progresión de castañar, encinar y robledal aclarados hacia formaciones más densas o las 388.08 has en donde el abandono de cultivos ha permitido la expansión de estas especies arbóreas que ya convivían con los usos agrícolas. En general se trata de comunidades mixtas que se disponen de manera dispersa entre cultivos actuales o abandonados. En estos casos se observa una progresiva ocupación de los terrenos adyacentes, ya sea de áreas de matorral o eriales originados por el abandono agrícola. 
Es, por lo tanto, la encina la que muestra una relativa expansión, con una evolución progresiva desde formaciones de matorral a encinar aclarado o desde encinares aclarados a encinares más densos, junto a otras dinámicas de menor relevancia. Estos datos muestran, en definitiva, que la encina es una especie en expansión en la Alpujarra Alta, que está recuperando su área potencial ocupada hasta hace poco por cultivos, a pesar de la acusada degradación que han sufrido los suelos, pero que incluso llega a invadir a veces el área potencial del robledal (Jiménez, 1991).

Por su parte, el hecho de que asistamos a un proceso de regeneración de las masas de robles que tiene como punto de partida los años 30 del siglo $\mathrm{XX}$, fecha en la que se produjo la última tala masiva, no anula la constatación de que los robledales que hoy se conservan en la Alta Alpujarra son relictos y se encuentran en un avanzado estado de degradación. Tan sólo el abandono, a partir de los años ochenta, de algunos cultivos de altura ubicados en el interior de las masas arboladas ha favorecido la generación del roble (331.04 has), a pesar de que ésta es difícil dada la alta sensibilidad de la especie a la degradación de los suelos una vez cultivados.

Con respecto a los castañares, hoy día constituyen el único reducto del conjunto de árboles frutales de regadío que se dio en el pasado, lo que no significa que las circunstancias actuales y futuras sean favorables a su conservación. De hecho el retroceso generalizado que sufre esta formación está ligado al deterioro de la red de acequias, consecuencia del abandono agrícola que sufre la comarca, que ha provocado una disminución de la humedad de los suelos y consecuentemente se han secado muchos de los ejemplares de castaño, especie que exige unas determinadas condiciones de humedad para su mantenimiento. En definitiva, su vocación agrícola se manifiesta en su propia evolución, que corre paralela al abandono de las tierras de cultivo. En este sentido la degradación es más acusada en el caso de las comunidades monoespecíficas (306.97 has).

Por último, cabría hablar de las transformaciones que sufren las escasas áreas cultivadas de secano, que tienden hacia el monocultivo arbóreo (García, 1999). Se trata de una evolución desde el secano herbáceo/leñoso hacia el secano exclusivamente leñoso, principalmente compuesto por almendros, que afecta a un total de 340.14 hectáreas. Este proceso es más intenso entre 1974 y 1987 y se localiza fundamentalmente en las laderas bajas y aterrazadas.

\section{Síntesis de los cambios en el paisaje: número de transformaciones y tendencias evolutivas}

El análisis pormenorizado de las principales dinámicas, ya sea por períodos o en el balance global, debe completarse con la cartografía de síntesis de todos los cam- 
bios ocurridos en el paisaje del área de estudio. Para facilitar su representación y comprensión, en este apartado se ha utilizado la leyenda reagrupada en nueve categorías, tal y como se definió en páginas anteriores. Este hecho implica, no obstante, que no se consideren las modificaciones en el seno de cada una de las grandes clases, por lo que las cifras de las áreas dinámicas son inferiores a las obtenidas a partir de la leyenda completa. En cualquier caso, los grandes cambios en el paisaje son visibles (ver CD adjunto).

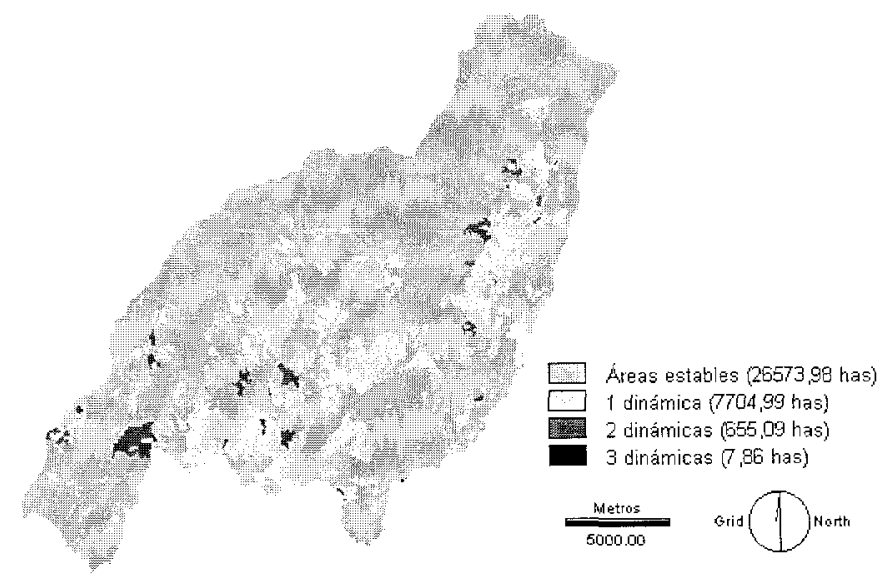

Figura 5. Número de transformaciones entre 1957-74-87-01 (Has) (Leyenda resumida). Fuente: Elaboración propia.

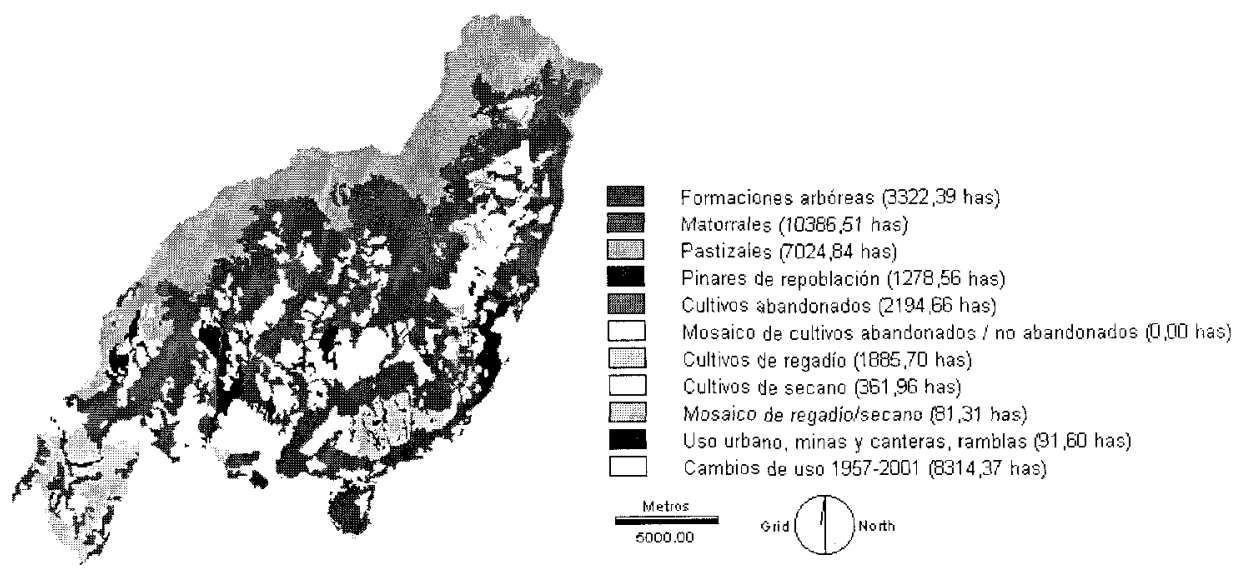

Figura 6. Áreas estables entre 1957 y 2001 (Has) (Leyenda resumida). Fuente: Elaboración propia. 
En primer lugar, la Figura 5 muestra el número de transformaciones que sufre cada una de las unidades espaciales de referencia (píxel) en las cuatro fechas analizadas. Destaca la significación superficial de las áreas estables, que suponen un $76.05 \%$ del total, y que se explican en gran medida por la aparente inmovilidad de las comunidades de los pisos crioro y oromediterráneo a la escala utilizada. En la Figura 6 se representan por categorías (según la leyenda resumida) las áreas que han permanecido estables en el periodo considerado.

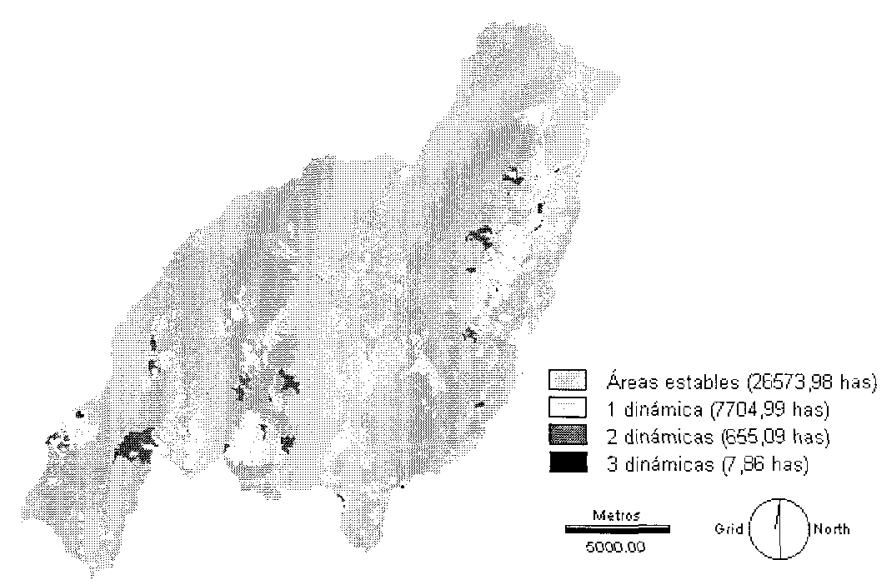

Figura 7. Areas dinámicas entre 1957 y 2001: tendencias evolutivas (Has) (Leyenda resumida). Fuente: Elaboración propia.

Respecto a las áreas que muestran algún tipo de transformación (Figura 5), la mayor parte $(22.05 \%$ del total) se ven afectadas tan sólo por un tipo de dinámica, frente al $1.87 \%$ que sufren dos transformaciones y el $0.02 \%$ con tres cambios sucesivos. La Figura 7 muestra el total de las áreas dinámicas entre 1957 y 2001, reagrupadas según las principales tendencias de cambio. Éstas han sido definidas tras el diagnóstico de todas las combinaciones resultantes de la superposición de los cuatro mapas de usos del suelo y vegetación con la leyenda reclasificada y pretenden expresar de manera sintética los ejes principales que definen la evolución temporal del paisaje del área de estudio.

\section{Conclusiones}

El análisis realizado en las páginas anteriores, centrado en la segunda mitad del siglo XX, debe enmarcarse en la tendencia evolutiva del paisaje de la mon- 
taña mediterránea, caracterizada por una disminución progresiva de la presión agrícola sobre el territorio, tan sólo alterada en los años 40 y 50 . En estos años incluso se roturaron nuevas tierras de difícil accesibilidad y bajo adversas condiciones naturales. Sin embargo, al inicio de la década de los sesenta, la despoblación, generalizada en las montañas españolas, provocó la reactivación del proceso de abandono.

En el conjunto de Sierra Nevada, la Alta Alpujarra constituye un ejemplo significativo de este intenso proceso de abandono, que afectó en primer lugar a las tierras de secano y a los regadíos de sierra, y a los regadíos permanentes de las laderas medias con posterioridad. Todo ello significó su transformación hacia formaciones de erial y contribuyó en gran medida a la homogeneización del paisaje de la montaña mediterránea. En la actualidad este paisaje agrícola está dominado por un proceso de "extensificación" y "naturalización" materializado en el protagonismo que adquieren los campos abandonados tanto antiguos como recientes, que muestran su tendencia a integrarse en las áreas potenciales de la vegetación natural.

En la Figura 7 se constata de una manera más evidente el proceso de "extensificación" que sufre la Alta Alpujarra. La mayor parte de la superficie que presenta un cambio de uso del suelo desde 1957 al 2001 está relacionada con el abandono y semiabandono de la agricultura (tanto de secano como de regadío), con la progresión natural de las comunidades de vegetación natural, ya sean herbáceas, arbustivas ("matorralización" en terrenos de erial y pastizal) o arbóreas (principalmente el encinar), o con la repoblación con coníferas.

Frente a estas cifras, la regresión de la vegetación natural o repoblada afecta a una superficie menor, pudiendo identificar varias causas. Por una parte, se trataría del retroceso de comunidades vegetales como la del castañar, que evidencia el proceso de abandono agrícola, u otras de carácter arbóreo y/o arbustivo, y también de los efectos de los incendios acaecidos en las últimas décadas (Camacho et al, 2002b). Además, el retroceso de las masas reforestadas puede deberse al "fracaso" de la tarea repobladora, que, a pesar de su eficacia, debería haber evitado el impacto que las especies foráneas crean en los ámbitos que las acogen (Jiménez, 1991), proceso que se ve confirmado por la tendencia natural del territorio a favorecer la regeneración espontánea de las especies autóctonas entre los pinos repoblados.

Junto a estas grandes tendencias, también son visibles cambios de escasa significación superficial, aunque no por ello menos relevantes en la dinámica paisajística actual y futura. Los nuevos regadíos o la transformación de secanos en cultivos regados (ligados a la introducción de nuevas especies de cultivo de mayor rentabilidad o a la ampliación del olivar de regadío en las laderas más bajas) (Camacho et al., 
2002a), son transformaciones detectadas en los últimos años que, al margen de las grandes tendencias y a pesar de afectar a enclaves dispersos, pueden ser indicativas de la búsqueda puntual de soluciones para frenar o invertir el proceso generalizado de abandono que, como se ha constatado en las páginas precedentes, ha dejado unas huellas evidentes en el paisaje del área de estudio.

\section{Notas}

1 «Dynamiques et modélisations de l'occupation du sol appliquées aux anthroposystèmes montagnards méditerranéens: Pyrénées - Sierra Nevada. Programme Environnement, Vie et Sociétés. C.N.R.S. Laboratorio GEODE UMR 5602, Universidad de Toulouse Le Mirail. Instituto de Desarrollo Regional, Universidad de Granada. 2000-2002.

"Sistemas de Información Geográfica y modelización de la dinámica paisajística de lá montaña mediterránea: Sierra Nevada y Pirineos Orientales franceses". Proyectos de I+D. MCYT. BLA2003-01499. Instituto de Desarrollo Regional, Universidad de Granada. Laboratorio GEODE UMR 5602, Universidad de Toulouse Le Mirail. 2003-2006.

\section{Bibliografía}

Bosque, J. (1969): Tradición y modernidad en las Alpujarras granadinas (Andalucía Oriental). XXI Congreso Geográfico Internacional, p 157-169. Madrid, CSIC.

Camacho, M.T. y Menor, J. (1997): Posibilidades de análisis y caracterización temporal y espacial mediante un Sistema de Información Geográfica en formato vectorial. Cuadernos Geográficos de la Universidad de Granada, 27, p. 197-217.

Camacho, M.T., García, P., Jiménez, Y., Menor, J. y Paniza, A. (2002a): La transformación de un paisaje de montaña: el proceso de abandono de la agricultura en la Alta Alpujarra granadina. XI Coloquio de Geografia Rural. Los espacios murales entre el boy y el mañana. AGE. Universidad de Cantabria, p. 547-559.
Camacho, M.T., García, P., Jiménez, Y., Menor, J. y Paniza, A. (2002b): La Alta Alpujarra granadina en la segunda mitad del siglo XX a través de la cartografía evolutiva de su paisaje: dinámica vegetal y repoblación forestal. XI Coloquio de Geografia Rural. Los espacios nurales entre el boy y el mañana. AGE. Universidad de Cantabria, p. 535-547.

Castro, R. y García, J. (1993): Confección de cartografía dinámica de la ocupación del suelo con SIG: municipio de Brea de Tajo. Madrid, II Congreso de la Asociación Española de Sistemas de Información Geográfica y Territorial (AESIGYT), p. 375-392.

Comas, D. et al. (1992): Evolución de los usos del suelo en la Alta Garrotxs entre 1975-1989. Madrid, I Congreso de la 
Asociación Española de Sistemas de Información Geográfica y Territorial (AESIGYT), p. 454-466.

Eastman, J.R. (2001): Idrisi32 release 2 Tutorial, Clarklabs, Worcester.

Eastman, J.R., Mckendry, J.E. y Fulk, M.A. (1995): Explorations in Geographic Information Systems technology. Change and time series analysis. United Nations Institute for Training and Research (UNITAR). Suiza, 119 pp.

García, P. (1999): La transformación del paisaje y la economía mural en la Alta Alpujarra Occidental. Granada, Universidad de Granada.

Jiménez, Y. (1991): Los Paisajes de Sierra Nevada. Granada, Universidad de Granada.

Martínez, J. y Romero, R. (1999): La dinámica de los usos del suelo en la conservación de un espacio protegido del sudoeste de la Comunidad de Madrid. En El territorio $y$ su imagen, vol I. AGE, Universidad de Málaga, p. 169, 177.

Martínez, J.E., Martín, J. y Román, S. (2000): Paisajes amenazados de la cuenca mediterránea. Aplicación del SIG en el análisis de la dinámica de usos del territorio (1956-1998) en la Vall de Gallinera (Alicante, España). Mediterránea. Serie de Estudios Biológicos, Epoca II, 17, Universidad de Alicante.

Otero, I. (1993): La aplicación del PC-ARC/INFO. Análisis del cambio paisajístico. Madrid, II Congreso de la Asociación Española de Sistemas de Información Geográfica $y$ Territorial (AESIGYT), p. 501-518.
Otero, I. (1999): Paisaje, teledetección y SIG: conceptos y aplicaciones. Madrid, Functación Conde del Valle de Salazar.

Paegelow, M. y Camacho, M.T. (1997): Dynamique spatio-temporelle de l'utilisation du sol en milieu montagnard méditerranéen. Etude comparative des Garrotxes (Pyrénées Orientales, France) et du Poqueira (Sierra Nevada, Espagne) En Le Temps de l'environnement. Presses Universitaires du Mirail, Toulouse, Francia,

Palacios, M. (1994): Sistemas de Información temporal: aplicación a la evaluación de los cambios ambientales en el valle medio del Jarama (Madrid). Mapping, 15, p. 29-31.

Peña, J. (en prensa): Análisis de los cambios de usos del suelo (1946-1999) en una cuenca semiárida (Agost, Alicante). Tesis de licenciatura. Universidad de Alicante.

Sancho, J., Bosque, J. y Moreno, F. (1993): La dinámica del paisaje: aplicaciones de un SIG raster al ejemplo de Arganda del Rey en las vegas de Madrid. Catastro, 18, p. 35-51.

Sancho, J., Bosque, J. y Moreno, F. (1993): Crisis and permanence of the traditional Mediterranean landscape in the central region of Spain. Landscape and Urban Planning, 23, p. 155-162.

Santos, J.M. y Borderías, M.P. (2001): Introducción al análisis medioambiental de un territorio. Madric, Universidad Nacional de Educación a Distancia.

Singh, R.B., Fox, J. y Himiyama, Y. (eds.) (2001): Land Use and Cover Change. Enfield, NH: Science Publishers. 
Tabla 1. Usos del suelo y vegetación de la Alta Alpujarra en 1957, 1974, 1987 y 2001 (Has).

\begin{tabular}{|c|c|c|c|c|}
\hline Leyenda de usos del suelo y vegetación & 1957 & 1974 & 1987 & 2001 \\
\hline 1. Encinar & 168,98 & 214,12 & 307,09 & 307,15 \\
\hline 2. Encinar con castanar & 47,20 & 55.31 & 55,31 & 55,31 \\
\hline 3. Encinar uclarado & 406,55 & 396,26 & 390,89 & 414,09 \\
\hline 4. Chaparal & 31,74 & 47,76 & 50,44 & 47,45 \\
\hline 5. Robledal & 717,13 & $1.227,81$ & $1.233,73$ & $1.140,14$ \\
\hline 6. Robledal con encinar & 514,36 & 515,54 & 515,54 & 515,54 \\
\hline 7. Robledal aclarado & 397,82 & & & 93,65 \\
\hline 8. Castanar & 320,62 & 315,26 & & \\
\hline 9. Cascañar con encinar y robledal & & 95,21 & 762,83 & 762,77 \\
\hline tañar con encinar, robledal y cultivos & 388,15 & 388,21 & & \\
\hline nar aclarado & & & 323,18 & 323,18 \\
\hline ñar aclaraclo con encinar y robledal aclarado & 374,50 & 279,47 & 10,10 & \\
\hline 13. V & 25,56 & 25,56 & 25,56 & 100,33 \\
\hline ra con pinar & 34,98 & 35,04 & 34,98 & 35,98 \\
\hline del encinar & 497,46 & 497,39 & 502,82 & 460,98 \\
\hline lediterráneo & $1.143,57$ & $1.193,89$ & $1.170,32$ & $1.161,77$ \\
\hline egetal & 97,52 & 126,76 & 128,20 & 128,20 \\
\hline diteráneos & 62,54 & 189,87 & 191,24 & 147,40 \\
\hline 19. Íc & 211,50 & 109,93 & 109,93 & 119,93 \\
\hline supramediterríneo & 129,76 & 157,13 & 173,28 & 168,42 \\
\hline 21. Ídem & & 14,90 & 14,90 & 14,90 \\
\hline 22. Mato & 92,59 & 92,66 & 93,65 & 93,65 \\
\hline 23. Id & 703,41 & 676,35 & 815,52 & 867,59 \\
\hline 24. Pio & $8.230,63$ & $7.561,14$ & $7.555,28$ & $7.627,86$ \\
\hline 25. Íde & 678,91 & 667,93 & 744,01 & 734,40 \\
\hline psicroxerófilo crioromediterráneo & $6.593,47$ & $6.626,27$ & $6.624,77$ & $6.624,77$ \\
\hline iles & 474,39 & 625,03 & 626,53 & 626,53 \\
\hline 28. $p$ & 463,04 & $1.352,58$ & $1.463,88$ & $1.552,42$ \\
\hline ión con encinar & 565,49 & 699,61 & 675,10 & 614,06 \\
\hline n encinar, robledal y castañar & 274,23 & 282,65 & 331,41 & 327,23 \\
\hline ción con menos del $50 \%$ de cobertura & 179,64 & 661,57 & 721,99 & 730,41 \\
\hline dos & 781,17 & $1.370,22$ & $1.735,99$ & $1.904,97$ \\
\hline los con matorral de degradación del robledal & 165,42 & 142,79 & & \\
\hline $34 . \mathrm{C}$ & $2.276,41$ & $2.141,98$ & $2.213,49$ & $2.147,15$ \\
\hline 35. C & 48,95 & 65,78 & 65,78 & 357,41 \\
\hline on herbiceos de reandín & 89,91 & & & 763,21 \\
\hline 37. Mosaico de & & & 485,49 & $1.061,38$ \\
\hline bandonados/no abandonados con leñosos de & & & 213,62 & 326,86 \\
\hline báceos & $3.095,11$ & $2.676,72$ & $2.012,41$ & 583,13 \\
\hline ceos con castañar & 34,11 & 34,17 & & \\
\hline 41. $\mathrm{C}$ & 876,44 & 920,15 & 921,46 & $1.040,93$ \\
\hline áceos/arbóreos & $1.075,91$ & 973,21 & 884,67 & 395,13 \\
\hline báceos/arbóreos con castanar & 28,68 & 28,68 & 22,63 & \\
\hline herbáceos & 765,39 & 213,19 & & \\
\hline heibáceos con robledal & 93,16 & 88,67 & & \\
\hline herbáceos/arbóreos & 499,76 & 94,78 & & \\
\hline 47. Cultivos de & 227,78 & 619,30 & 495,40 & 365,83 \\
\hline 48. Mosaico de reg & 441,09 & 278,97 & 114,73 & 31,74 \\
\hline 49. Mosaico de regadio/secano herbáceos con cultivos abandonados & 338,39 & & & 55.74 \\
\hline 50. Mosaico de regadio/secano herbáceos con robledal & 180,45 & 43,71 & - & \\
\hline & 83,24 & 84,12 & 86,80 & 86.36 \\
\hline as y canteras & 10,41 & 29,87 & 29,87 & 29,87 \\
\hline 53. Ramblas & 4,36 & 4,36 & 7,04 & 7,04 \\
\hline TOTAL & $34.941,88$ & $34.941,88$ & $34.941,88$ & $34.941,88$ \\
\hline
\end{tabular}

Fuente: Elaboración propia. 
Tabla 2. Principales dinámicas de los usos del suelo y vegetación entre 1957-74, 1974-87 y 1987-2001 (Has).

\begin{tabular}{|c|c|c|c|}
\hline PRINCIPALES DINÁMICAS (Antigua/nueva categoria) & $1957-74$ & $1974-87$ & $1987-01$ \\
\hline Progresión del robledal $(7 / 5)$ & 331,16 & - & - \\
\hline Regresión del robledal $(5 / 7)$ & & - & 93,66 \\
\hline Abandono de cultivos entre castañar y otras formaciones arbóreas (10/9) & & 388,09 & - \\
\hline Progresión del castañar, encinar y robledal aclarados (12/9) & 95,21 & 279,47 & - \\
\hline Regresión del castañar (8/11) & - & 315,26 & - \\
\hline Progresión del matorral de degradación del robledal en cultivos abandonados $(33 / 23)$ & - & 101,45 & - \\
\hline Transformación de cultivos abandonados con piornal a piornal $(34 / 24)$ & 38,41 & 19,52 & 107,62 \\
\hline Repoblación con pinares sobre piornal oronediterráneo $(24 / 28)$ & 412,22 & 57,80 & 35,17 \\
\hline Progresión del pinar de repoblación (31/28) & 94,47 & & 269,06 \\
\hline Regresión del pinar de repoblación (28/31) & 13,09 & 18,77 & 215,25 \\
\hline Repoblación pinares (<50\%) sobre piornal oromediterráneo (24/31) & 367,58 & 41,53 & \\
\hline Abandono de mosaico de herbáceos/ arbóreos de regadio semi-abandonados (37/32) & & & 241,37 \\
\hline Abandono de cultivos de regadío herbáceos $(39 / 32)$ & 213,00 & 144,41 & 271,80 \\
\hline Abandono de cultivos de secano herbáceos (44/32) & 85,67 & 166,61 & \\
\hline Abandono de mosaico de regadío/ secano herbáceo (48/32) & 153,76 & 94,53 & 33,36 \\
\hline Abandono de mosaico de regadio/ secano herbáceos semi-abandonados (49/32) & 295,99 & & \\
\hline Abandono de cultivos de secano herbáceos y progresión del piornal ( $44 / 34)$ & 274,67 & 27,25 & - \\
\hline Progresiốn del castañar y otras formaciones arbóreas en cultivos abandonados $(32 / 35)$ & - & - & 213,81 \\
\hline Semi-abandono de cultivos de regadío herbáceos $(39 / 36)$ & - & & 760,84 \\
\hline Semi-abandono de regadios herbáceos y transformación a herbáceos/ arbóreos (39/37) & - & 367,64 & 268,74 \\
\hline Semi-abandono de regadíos herbăceos/arbóreos (42/37) & - & 114,79 & 499,64 \\
\hline Semi-abandono de secanos arbóreos $(47 / 38)$ & - & 213,62 & 73,02 \\
\hline Transformación de secanos herbáceos/arbóreos a arbóreos (46/47) & 394,08 & 73,02 & \\
\hline TOTAL Principales dinámicas & $2.769,31$ & $2.423,76$ & 3083,34 \\
\hline Otras dinámicas & $2.316,76$ & $1.070,92$ & 639,74 \\
\hline TOTAL ÁREAS DINÁMICAS & $5.086,07$ & $3.494,68$ & $3.723,08$ \\
\hline TOTAL ÄREAS ESTABLES & $29.855,81$ & $31.447,20$ & $31.218,80$ \\
\hline TOTAL & $34.941,88$ & $34.941,88$ & $34.941,88$ \\
\hline
\end{tabular}

Fuente: Elaboración propia.

Tabla 3. Principales dinámicas de los usos del suelo y vegetación entre 1957-2001 (Has).

\begin{tabular}{|lr|}
\hline PRINCrPALES DINÁMICAS (Antigua/nueva categoria) & $\mathbf{1 9 5 7 - 0 1}$ \\
\hline Progresión del robledal (7/5) & 331,04 \\
Abandono de cultivos entre castañar y otras formaciones arbóreas (10/9) & 388,09 \\
Progresión del castañar, encinar y robledal aclarados (12/9) & 374,05 \\
Regresión del castañar (8/11) & 306,97 \\
Repoblación con pinares sobre piornal oromediterráneo (24/28) & 470,77 \\
Repoblación sobre cultivos abandonados con piornal (34/28) & 435,60 \\
Repoblación pinares (<50\%) sobre piornal oromediterráneo (24/31) & 456,12 \\
Abandono de cultivos de regadío herbáceos (39/32) & 694,87 \\
Abandono de mosaico de regadio/ secano herbáceos semi-abandonados (49/32) & 310,02 \\
Abandono de cultivos de secano herbáceos y progresión del piornal (44/34) & 351,00 \\
Semi-abandono de cultivos de regadío herbáceos (39/36) & 739,33 \\
Semi-abandono de regadíos herbáceos y transformación a herbáceos/ arbóreos (39/37) & 468,90 \\
Semi-abandono de regadíos herbáceos/ arbóreos (42/37) & 484,11 \\
Transformación de secanos herbáceos/ arbóreos a arbóreos (46/47) & 340,14 \\
TOTAL Principales dinámicas & $6.151,01$ \\
Otras dinámicas & $4.027,42$ \\
TOTAL ÁREAS DINÁMICAS & $10.178,43$ \\
TOTAL ÁREAS ESTABLES & $24.763,45$ \\
TOTAL & $34.941,88$ \\
\hline
\end{tabular}

Fuente: Elaboración propia. 